\title{
Widened Learning of Bayesian Network Classifiers
}

\author{
Oliver R. Sampson ${ }^{(凶)}$ and Michael R. Berthold \\ Chair for Bioinformatics and Information Mining, \\ Department of Computer and Information Science, \\ University of Konstanz, Konstanz, Germany \\ oliver.sampson@uni-konstanz.de
}

\begin{abstract}
We demonstrate the application of Widening to learning performant Bayesian Networks for use as classifiers. Widening is a framework for utilizing parallel resources and diversity to find models in a hypothesis space that are potentially better than those of a standard greedy algorithm. This work demonstrates that widened learning of Bayesian Networks, using the Frobenius Norm of the networks' graph Laplacian matrices as a distance measure, can create Bayesian networks that are better classifiers than those generated by popular Bayesian Network algorithms.
\end{abstract}

\section{Introduction}

Widening [2,18] formalizes a method for executing a greedy learning algorithm in parallel while using diversity to guide the parallel refinement paths through a hypothesis ${ }^{1}$ space. This enables the system as a whole to avoid local optima and potentially find better models than the greedy learning algorithm would otherwise find. Previous work $[13,29]$ has demonstrated its viability on real world algorithms. This work builds on that with an application to the superexponentially-sized [28] hypothesis space of learning Bayesian Networks. Bayesian Networks [26] are probabilistic graphical networks, which describe relationships of conditional dependence between the features of a dataset. Perhaps the best known of these graphical networks is the network defined by the NAÏVE BAYES algorithm [11,23]. This paper describes the application of Widening to the learning of Bayesian Networks for use as classifiers.

The ultimate goal of Widening is not just to provide better solutions using parallel resources, but to provide better solutions in the same time or less than the canonical greedy algorithm. To enable this, communication-free Widening would allow the model refinement paths, separated by some measure of diversity, to be followed through the solution space until some stopping criterion is met. The difficulty in that effort has been finding a suitable measure of distance, i.e.,

\footnotetext{
${ }^{1}$ We freely mix the use of "solution space" and "hypothesis space" throughout this paper, referring essentially to the same space, but drawing attention to whether it is the evaluation of the hypothesis or the hypothesis itself that is important.
} 
diversity. Here, we show that the Frobenius Norm of Bayesian Networks' graph Laplacians is a useful measure of diversity for comparing Bayesian networks in the Widening framework, albeit not in a communication-free framework.

\section{Background}

\subsection{Learning and Scoring Bayesian Networks}

A Bayesian network, $B$, derived from a dataset, $\mathcal{D}$, is a triple, $\langle\mathcal{X}, G, \Theta\rangle$, where $\mathcal{X}$ is the set of features or random variables in the dataset, $G$ is a directedacyclic-graph (DAG), and $\Theta$ is the set of conditional probability tables (CPT) for the features in $\mathcal{X}$. The graph $G=(\mathcal{X}, \mathcal{E})$, is an ordered pair, where each node, $X \in \mathcal{X}$, is a feature from the dataset and where each edge, $E=\left\{X_{i}, X_{j}\right\} \in \mathcal{E}$, is directed according to the dependency of one feature on another.

There are four general categories of algorithms for learning Bayesian networks: search-and-score, constraint-based, hybrid [19] and evolutionary algorithms [20]. Search-and-score methods such as K2 [9] and GrEedy EQUivaLENCE SEARCH (GES) [8] rely on heuristics to sequentially add, remove, or change the direction of the edges in the graph, $G$, to which a scoring method is applied. Edges that improve the score are kept in the graph for the next iteration of add, delete, or change. Constraint-based methods such as PC ALGORITHM [32] or CBL [7] rely on some assumptions about the dependency relationships of the features, from which a partially-directed-acyclic-graph is generated. This "skeleton" of a graph describes the neighbors of each of the feature nodes within the graph, but not necessarily the direction of the edges between the nodes. After determining the skeleton, search-and-score methods are used to find better networks, i.e., networks with a higher score, by flipping the direction of the edges and re-evaluating the score. Hybrid methods such as MAX-Min Hill-Climbing [35] incorporate techniques from both the search-and-score and the constraint-based methods. Algorithms based on evolutionary techniques randomly change and combine networks and evaluate them with a fitness function.

Several scoring functions have been proposed for the use of learning Bayesian networks. For an extensive overview and comparison, the reader is referred to [6]. Scoring functions can be grouped into two categories, Bayesian and informationtheoretic. Bayesian scoring methods calculate the posterior probability distribution based on the prior probability distribution conditioned on $\mathcal{D}$. Some examples of Bayesian scoring functions are K2 [9], Bayesian Dirichlet (BD) [17], BD with equivalence assumption (BDe) [9], and $\mathrm{BD}$ with equivalence and uniform assumptions (BDeu) [5].

Information-theoretic score functions are based on Shannon entropy and the amount of compression possible for a Bayesian network. The Log-Likelihood (LL) score is based on the logarithm of the likelihood of $\mathcal{D}$ given $B$, i.e., $\log (P(\mathcal{D} \mid B)$ ). The LL score is better, in general, for complete networks, and for this reason alternate scoring functions have been proposed that penalize the LL according to some factor. The Minimum Description Length [34] (MDL), the Akaike Information Criterion [1] (AIC), and Bayesian Information Criterion [30] (BIC) (roughly 


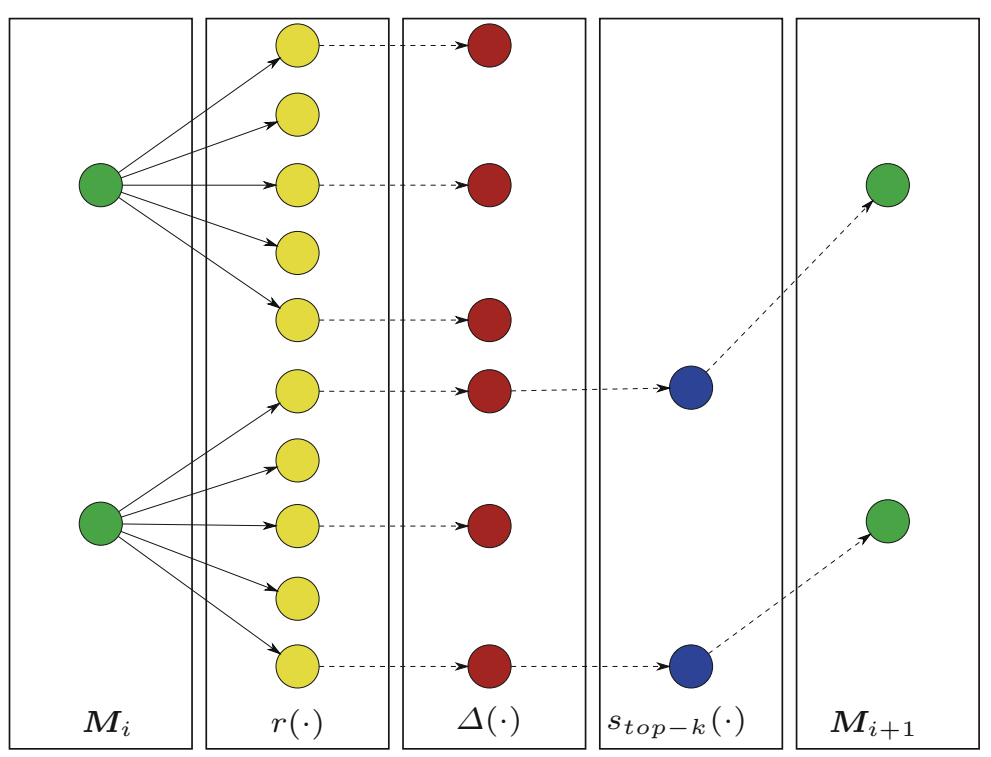

Fig. 1. Widening. Each $m_{k, i} \in \boldsymbol{M}_{i}$ (green) is refined to five models (yellow). In each of these sets, the three most diverse from another are determined (red). The two best performing models (blue) are selected and used for the next iteration, $m_{k, i+1} \in \boldsymbol{M}_{i+1}$ (green). (Color figure online)

equivalent to MDL) all adjust the LL by different proportions of the network complexity.

Less commonly, and more often associated with evolutionary algorithms such as $[27,31]$, the performance of the networks as a classifier is used as the scoring, i.e., fitness, function. In this vein, the work described in this paper also uses accuracy as the scoring function.

When used as a classifier, the relevant portion of the network contains the parents of the target node, the children of the target node, and the children's other parents. This is termed the Markov blanket [26, p. 97].

\section{$2.2 \quad$ Widening}

The Widening framework [2,18] (See Fig. 1.) describes a general process for improving greedy learning algorithms where models, $m \in \mathcal{M}$, are iteratively refined and scored in parallel. Each refinement path follows a different route through the hypothesis space. The models at each refinement step are separated using a diversity measure, $\Delta$, which enforces a distance between the models' respective refinement paths.

More formally, a refinement operator, $r(\cdot)$, applied to a model, $m$, generates a set of models, $\boldsymbol{M}^{\prime}$, from the set of all possible models, $\mathcal{M}$, in the hypothesis space. A selection operator, $s(\cdot)$, when applied to a set of models, selects a subset according to a performance metric. In Widening's most rudimentary form, the best $k$ performing models from a refinement step are selected, $s_{\text {top }-k}\left(\boldsymbol{M}^{\prime}\right)$, which in turn are further refined and selected until a stopping criterion is met. The $s_{\text {top }-k}(\cdot)$ operator has a similarity to a BEAM SEARCH, [22] but instead of a 
selection operator based solely on performance, the selection is also based on diversity, due to the refinement operator.

\subsection{Related Work}

Learning Bayesian Networks for classification, either by modifying networks created by NAÏVE BAYES or by the generation of networks through completely different methods, is a very active research area. An excellent survey can be found in [4]. In [14], Friedman et al. describe Tree Augmented NaÏVE Bayes NETwORK (TAN) where edges are added between child nodes of a Naïve Bayes network in a greedy search using the MDL scoring function, and whose structure is limited to that of a tree. The authors also describe learning an "unrestricted" Bayesian Network Augmented NaÏve Bayes (BAN), but these networks do not include networks with nodes as parents for the target nodes, but rather just more complex relationships among the child nodes. Cheng et al. in [7] describe an algorithm (CBL) for finding General Bayesian Networks (GBN) based on conditional independence tests using Mutual Information (MI).

In [25] Nielsen et al. present K-Greedy Equivalence SEArCH (KES) which is a modification to the GES, where a random subset of models from the entire set is chosen and evaluated. They describe this as a method specifically to avoid the local optima encountered by GES in [8].

$\mathrm{Su}$ and Zhang describe in [33] what they call Full Bayesian Networks (FBN), which are TANs where all child nodes of the target are connected to a maximal subset of the other child nodes based on an ordering using MI. This structure is in turn used to learn a Decision Tree-like structure for learning CPT-Trees.

The work presented here is similar to the TAN in [14], in that we perform a greedy search for better networks starting with a network generated by NAÏvE BAYES. It is similar to the work in [25], in that a subset of models is chosen and evaluated specifically to avoid local optima. It differs from these two, in that (1) any configuration of Bayes Network is allowed, (2) diversity between networks rather than randomness is used to select models, and (3) classification accuracy is employed for the scoring function.

\section{$3 \quad$ Widened Bayesian Networks}

\subsection{Application of the Widening Framework}

The simplest search-and-score method (Hill-Climbing or GREEDy SEARCH) refines a Bayesian Network model by changing a randomly or heuristically chosen edge, $E$, and scores the network according to one of the scoring functions discussed in Sect. 2.1. The algorithm greedily keeps the changed edge if it improves the score. Using the Widening notation, the greedy search-and-score method is $B_{i+1}=s_{\text {top }-k=1}\left(r\left(B_{i}\right)\right)$, where $i$ refers to the current search-and-score iteration. The process stops when no further improvement is seen.

The application of Widening to this process is to refine a set of different Bayesian networks at each stage, $\boldsymbol{B}_{i+1}=r\left(\boldsymbol{B}_{i}\right)$. Each model is refined to a 
number, $l$, of refinements. From this set, $k$ models are selected by the selection operator, $s_{\text {top }-k}(\cdot) . k \times l$ models are generated during each refinement iteration, with the exception of the initial one. Additionally, the application of a diversity measure, $\Delta$, is used by the refinement operator, and therefore notated as $r_{\Delta}(\cdot)$. The refinement operator ensures that the models are different enough to explore disparate regions of the hypothesis space.

Scoring Bayesian Networks by using classification accuracy is common only with the evolutionary algorithms, even though, for example, Friedman et al. in [14] explicitly say that one of the reasons that their TAN ALGORITHM did not always provide superior solutions was that the structural score may not have been a good analog for the use of the network in its role as a classifier.

In summary, each step in the top- $k$ Widening process is described as

$$
\boldsymbol{B}_{i+1}=s_{\text {top }-k}\left(r_{\Delta}\left(\boldsymbol{B}_{i}\right)\right)
$$

\subsection{Refinement Operator}

The refinement operator creates a list of all possible pairs of nodes, i.e., all possible edges. Each edge is compared with the current model and up to two additional models are created based on the edge. (See Fig. 2.)

1. If it is possible to add the edge to the initial model (Fig. 2a), i.e., its presence would not contravene the definition of DAG by creating a loop, it is added. (See Fig. 2f.)

2. If it is present in the model, it is removed. (See Figs. $2 b$ and d.)

3. If it is present in the model, and the reversal of its direction would not create a loop, it is reversed. (See Figs. 2c and e.)

A distance matrix of all distances between network model pairs is then calculated.

\subsection{Diversity}

There are a variety of measures for comparing two labeled DAGs. Early experiments indicated that the Hamming distance [16] does not measure diversity in a way that scales well to larger networks. For this work, we have chosen the Frobenius Norm of the difference between the graphs' Laplacian matrices. The Frobenius Norm is sometimes referred to as the Euclidean norm and provides a "measure of distance on the space of matrices". [15] The Frobenius Norm for a matrix, $A \in \mathbb{R}^{m \times n}$ is defined as

$$
\|A\|_{F}=\sqrt{\sum_{i=1}^{m} \sum_{j=1}^{n}\left|a_{i j}\right|^{2}[15]}
$$

where, $a_{i j}$ are elements of matrix $A$. 


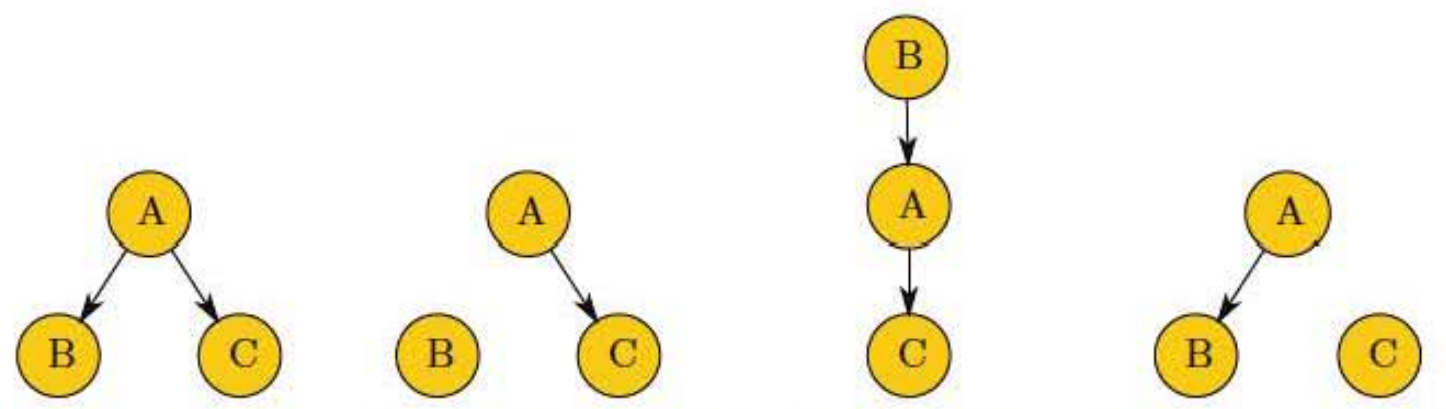

(a) Initial model. (b) $A \rightarrow B$ removed. (c) $A \rightarrow B \Rightarrow B \rightarrow A$ (d) $A \rightarrow C$ removed.
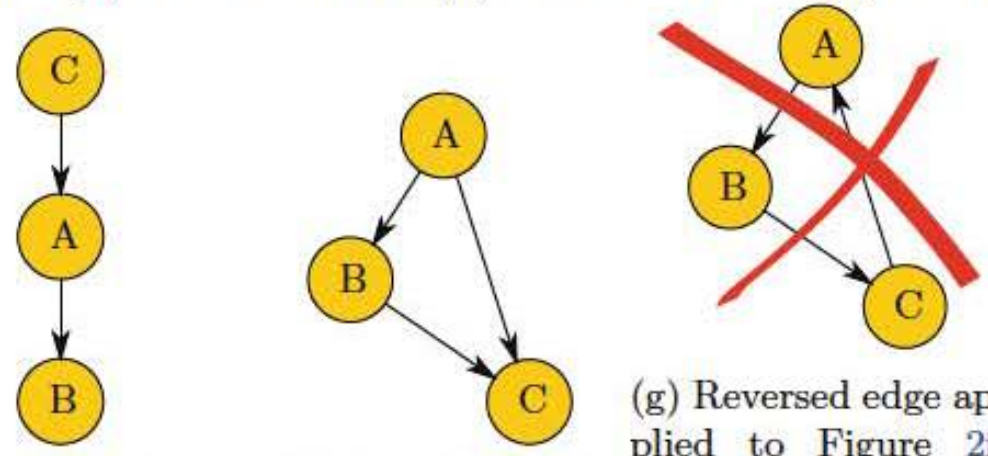

(g) Reversed edge applied to Figure 2f.

(e) $A \rightarrow C \Rightarrow C \rightarrow A$

(f) $B \rightarrow C$ added.

Disallowed; not DAG.

Fig. 2. Example possible refinements for the three edges $\{\langle A, B\rangle,\langle A, C\rangle,\langle B, C\rangle\}$

The Laplacian matrix of a graph is given by the formula $L=D-A$, where $D$ is the out-degree matrix, and A is the adjacency matrix. Here we use the Frobenius Norm of the difference of each pair of Bayesian networks' Laplacian matrices, i.e.,

$$
\Delta_{\text {Frobenius }}=\left\|L_{B_{p}}-L_{B_{q}}\right\|_{F}: B_{p}, B_{q} \in B_{i}
$$

and $\boldsymbol{B}_{i}$ is the set of refined Bayesian networks from Eq. 1 .

The P-Dispersion ProBlem describes selecting a subset of points from a larger set, where the subset's minimum pairwise distances are maximized. There are several diversity measures used commonly with the P-DISPERSION PROBLEM, including sum and min-sum. p-dispersion-sum simply maximizes the sum of the distances between any two points in the subset, whereas $p$-dispersionmin-sum maximizes the sum of the minimum distances between two points. p-dispersion-sum has the property of pushing the resultant subset to the margins of the original set, whereas the subset derived using $p$-dispersion-min-sum is more representative of the dataset as whole [24]. Because of this property, and based on the results in [29], we favor p-dispersion-min-sum as the diverse subset selection method.

Definition 1 p-dispersion-min-sum. ${ }^{2}$ Given a set $\boldsymbol{B}=\left\{B_{1}, \cdots, B_{n}\right\}$ of $n$ distinct Bayesian networks and $p$, where $p \in \mathbb{N}$ and $p \leq n$, and a distance

${ }^{2}$ In this application, it would be correctly termed " $l$-dispersion-min-sum," but the notation is written here as " $p$ " to be consistent with the literature. 
measure $d\left(B_{i}, B_{j}\right): B_{i}, B_{j} \in \boldsymbol{B}$ between Bayesian networks $B_{i}$ and $B_{j}$, the p-diversity-min-sum problem is to select the set $\hat{\boldsymbol{B}} \subseteq \boldsymbol{B}$, such that

$$
\begin{aligned}
\hat{\boldsymbol{B}} & =\underset{\substack{\boldsymbol{B}^{\prime} \subseteq \boldsymbol{B} \\
\boldsymbol{B}^{\prime} \mid=p}}{\operatorname{argmax}} f\left(\boldsymbol{B}^{\prime}\right), \text { where } \\
f\left(\boldsymbol{B}^{\prime}\right) & =\sum_{i=1}^{p} \min _{1 \leq i, j \leq n, i \neq j} d\left(B_{i}, B_{j}\right): B_{i}, B_{j} \in \boldsymbol{B}[24]
\end{aligned}
$$

The P-Dispersion PROBlem is known to be $\mathcal{N} \mathcal{P}$-complete, and when adjusting the diversity criterion to be min-sum, the problem is $\mathcal{N} \mathcal{P}$-hard [12].

\subsection{Selection Operator}

The selection operator presented in this work is simply the performance metric of the Bayesian network as a classifier, similar to that of [31]. When compared to the scoring methods described in Sect. 2.1, this has the advantage of being directly related to the network's use as a classifier, and networks that perform poorly as classifiers are eliminated from the refinement paths. The calculation for determining the target winner is similar to that of NAÏVE BAYES, except the probabilities of the parents of the target node and of the other parents of the target's child nodes are considered.

$$
\hat{C}=\underset{j=1, \ldots,|C|}{\operatorname{argmax}} P\left(C_{j}, \boldsymbol{X}_{m}\right)=\underset{j=1, \ldots,|C|}{\operatorname{argmax}} P\left(C_{j} \mid \boldsymbol{p a}(C)\right) \prod_{i=1}^{m} P\left(x_{i} \mid \boldsymbol{p a}\left(x_{i}\right)\right)[4]
$$

where $\boldsymbol{X}_{m} \subseteq \mathcal{X}$ is the subset of features contained in the Markov-blanket of the target node, $C$, and $\boldsymbol{p a}(\cdot)$ is the set of parents of a child of $C$ in the Bayesian network.

\section{Experimental Results}

The experiments were performed in KNIME Analytics Platform [3]. The datasets from the UCI Machine Learning repository ${ }^{3}$ [21] were discretized using the LUCS-KDDN software. ${ }^{4}$ Unlike algorithms such as K2 or CBL, no assumptions were made concerning the ordering of the features within the dataset. Datasets with missing values or continuous values were not considered, because we are interested in testing the Widened learning process and not the robustness of the algorithm to various data types. The refinement operator placed no restrictions on the number of parents a node may have. The stopping criterion was set to stop the iterations when improvement in the best model compared to its performance in the previous iteration was less than $0.01 \%$. The records in the datasets were shuffled between each widening trial of a different breadth and width.

\footnotetext{
3 http://archive.ics.uci.edu/ml/.

${ }^{4}$ http://www.csc.liv.ac.uk/ frans/KDD/Software/LUCS_KDD_DN/.
} 
Table 1. Accuracy $(\mu \pm 2 \sigma)$ comparison of all tested algorithms with 5-fold crossvalidation.

\begin{tabular}{l|r|r|l|l|l|l|l}
\hline Dataset & $|\mathcal{D}|$ & $|\mathcal{X}|$ & $|C|$ & WIdEnED BAYEs & MMHC & TABU & HiLL-Climbing \\
\hline ecoli & 336 & 7 & 8 & $\mathbf{0 . 7 4 7} \pm \mathbf{0 . 0 3 2}$ & $0.430 \pm 0.123$ & $0.593 \pm 0.119$ & $0.647 \pm 0.057$ \\
\hline flare & 1389 & 10 & 9 & $\mathbf{0 . 8 4 3} \pm \mathbf{0 . 0 1 5}$ & $\mathbf{0 . 8 4 3} \pm \mathbf{0 . 0 1 3}$ & $\mathbf{0 . 8 4 3} \pm \mathbf{0 . 0 1 3}$ & $\mathbf{0 . 8 4 3} \pm \mathbf{0 . 0 1 3}$ \\
\hline glass & 214 & 9 & 7 & $\mathbf{0 . 6 4 9} \pm \mathbf{0 . 1 3 7}$ & $0.457 \pm 0.151$ & $0.564 \pm 0.133$ & $0.536 \pm 0.111$ \\
\hline nursery & 12960 & 8 & 8 & $\mathbf{0 . 9 3 5} \pm \mathbf{0 . 0 4 7}$ & $0.570 \pm 0.150$ & $0.621 \pm 0.214$ & $0.632 \pm 0.246$ \\
\hline pageBlocks & 5473 & 10 & 5 & $0.898 \pm 0.015$ & $\mathbf{0 . 9 1 3} \pm \mathbf{0 . 0 1 1}$ & $\mathbf{0 . 9 1 3} \pm \mathbf{0 . 0 0 4}$ & $0.910 \pm 0.023$ \\
\hline pima & 768 & 8 & 2 & $0.710 \pm 0.068$ & $0.721 \pm 0.136$ & $\mathbf{0 . 7 5 7} \pm \mathbf{0 . 0 9 8}$ & $0.736 \pm 0.143$ \\
\hline wavef orm & 5000 & 21 & 3 & $\mathbf{0 . 7 9 0} \pm \mathbf{0 . 0 2 5}$ & $0.342 \pm 0.014$ & $0.619 \pm 0.020$ & $0.620 \pm 0.021$ \\
\hline wine & 178 & 13 & 3 & $\mathbf{0 . 9 3 9} \pm \mathbf{0 . 0 9 1}$ & $0.746 \pm 0.150$ & $0.798 \pm 0.116$ & $0.747 \pm 0.184$ \\
\hline
\end{tabular}

The initial state could be any network configuration that satisfies the definition of a DAG, including a network without any edges. Because our effort is to prove the ability of Widening to find superior solutions to traditional greedy methods, we chose a Naïve Bayes configuration, where all of the non-target features are dependent on the target variable, as the initial state. This was a pragmatic decision in the sense that finding a network out of all possible networks that is tuned to the target node is impractical. Additionally, NÄ̈vE BAYES performs remarkably well given its simplicity for a large number of datasets and is a measuring stick for many new algorithms.

We tested eight datasets, ecoli, flare, glass, nursery, pageBlocks, pima, waveform and wine against three standard Bayesian Network learning algorithms, MaX-Min Hill-Climbing (MMHC), TABu, and Hill-Climbing, from the $\mathbf{R}$ bnlearn ${ }^{5}$ package, version 3.8.1. MMHC and Hill-Climbing used parameters test $=\mathrm{mi}$, restart $=100$, and perturb $=100$. These values were chosen experimentally as values that provide good results for all datasets.

WIDENED BAYESIAN NETWORKS (WBN) significantly outperformed the other three reference implementations in five of the eight datasets, tied in one, and performed slightly worse in two (Table 1 ).

The results in Fig. 3 show a two responses to Widening. In general, with Widening we expect a gradual improvement of average performance with the width, i.e., the number of parallel paths in the solution space. Additionally, we expect a decrease in the variance of the results as the many paths push themselves towards better solutions. ecoli, glass, nursery, pima, waveform, and wine show this behavior nicely. pageBlocks and flare demonstrate how some solution space topologies cannot be explored with the refine-and-select process presented here, even though the results for the comparison algorithms for flare indicate that the resultant Bayesian network is a best fit. The nonresponsive nature of pageBlocks however, invites further research into other refining-and-select strategies and/or diversity measures.

\footnotetext{
${ }^{5}$ http://www.bnlearn.com/.
} 


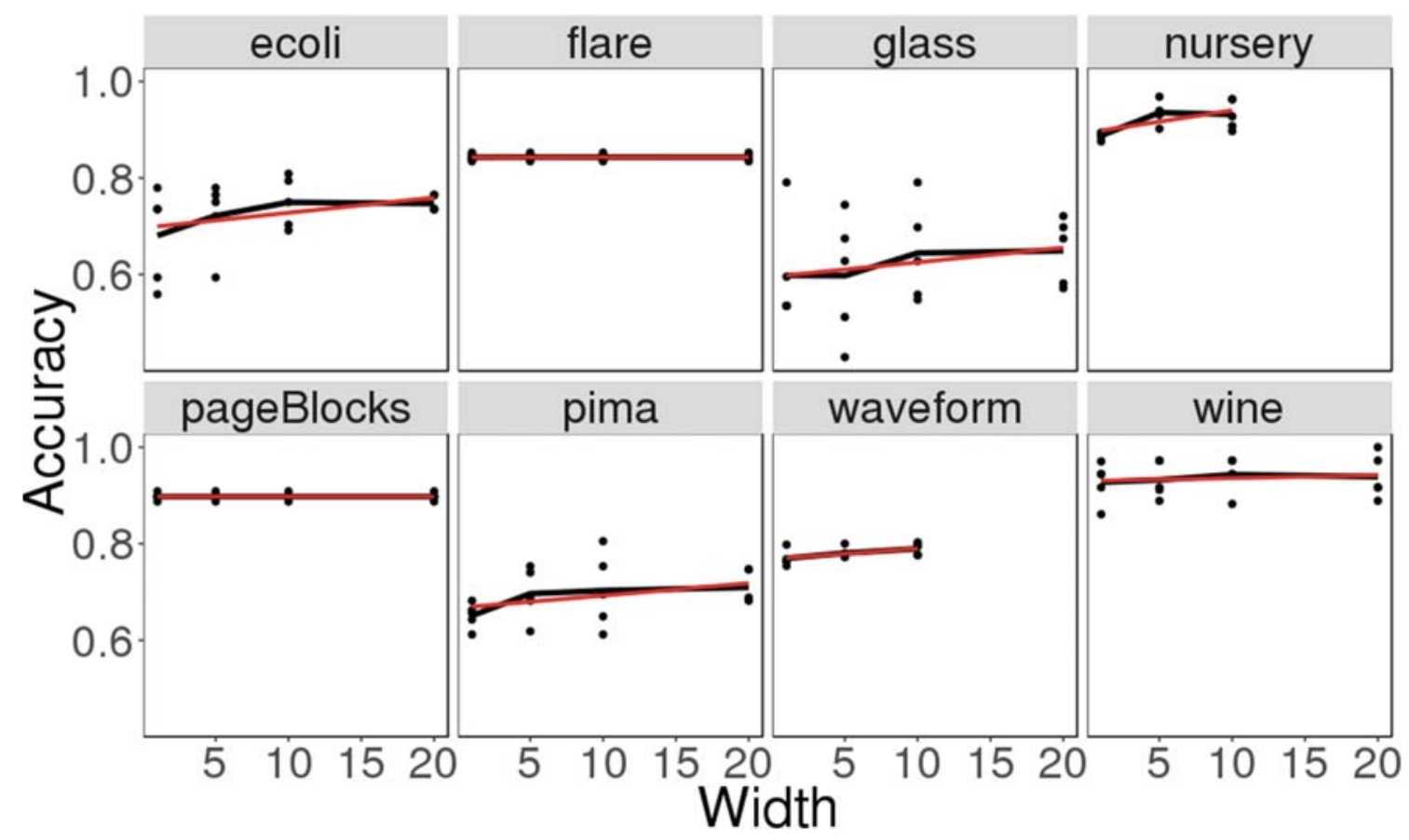

Fig. 3. Widened Bayesian Networks accuracy progression verses width with 5 -fold cross-validation.

\section{Conclusion and Future Work}

This paper demonstrates the successful initial application of Widening to learning Bayesian Networks for use classifiers and demonstrates classification scoring techniques with the search-and-score greedy heuristic. The technique was able to find superior solutions when compared to standard Bayesian Network learning algorithms from the $\mathbf{R}$ bnlearn package. Although the results are similar or superior to established Bayesian Network learning algorithms on some datasets, the execution time does not meet the specified goal of finding better solutions in the same time or less as the greedy algorithm. The primary impediment to this goal, as it is demonstrated here, is the use of p-dispersion-min-sum for finding a maximally diverse subset of networks for refinement. Methods that allow for diverse subsets to be calculated without communication between the parallel workers would be better. (See [18] for details.) Additionally, the refinement operator considers the entire space of possible networks, where only the refinements to the Markov blanket are actually necessary. Significantly, the use of the Frobenius Norm of the difference of the Bayesian networks's graph Laplacians is very encouraging and suggests further research into distance measures based on graph features such as those derived from Spectral Graph Theory. Experiments with alternate starting states based on conditional information, in a manner similar to the PC Algorithm and CBL, or constraint-based algorithms like Incremental Association or HITON, or even to those claiming to find the exact network structure [10] could also be promising. 


\section{References}

1. Akaike, H.: A new look at the statistical model identification. IEEE Trans. Autom. Control 19(6), 716-723 (1974)

2. Akbar, Z., Ivanova, V.N., Berthold, M.R.: Parallel data mining revisited. better, not faster. In: Hollmén, J., Klawonn, F., Tucker, A. (eds.) IDA 2012. LNCS, vol. 7619, pp. 23-34. Springer, Heidelberg (2012). doi:10.1007/978-3-642-34156-4_4

3. Berthold, M.R., Cebron, N., Dill, F., Gabriel, T.R., Kötter, T., Ohl, P., Sieb, C., Thiel, K., Wiswedel, B.: KNIME: the konstanz information miner. In: Preisach, C., Burkhardt, H., Schmidt-Thieme, L., Decker, R. (eds.) Data Analysis, Machine Learning and Applications. Studies in Classification, Data Analysis, and Knowledge Organization, pp. 319-326. Springer, Heidelberg (2007)

4. Bielza, C., Larranaga, P.: Discrete Bayesian network classifiers: a survey. ACM Comput. Surv. (CSUR) 47(1), 5 (2014)

5. Buntine, W.: Theory refinement on Bayesian networks. In: Proceedings of the Seventh Conference on Uncertainty in Artificial Intelligence, pp. 52-60. Morgan Kaufmann Publishers Inc., Los Angeles (1991)

6. Carvalho, A.M.: Scoring functions for learning Bayesian networks. Technical report INESC-ID Technical report 54/2009, Instituto superior Téchnico, Technical University of Lisboa, April 2009

7. Cheng, J., Bell, D.A., Liu, W.: An algorithm for Bayesian belief network construction from data. In: Proceedings of AI \& STAT 1997, pp. 83-90 (1997)

8. Chickering, D.M.: Optimal structure identification with greedy search. J. Mach. Learn. Res. 3, 507-554 (2002)

9. Cooper, G.F., Herskovits, E.: A Bayesian method for the induction of probabilistic networks from data. Mach. Learn. 9(4), 309-347 (1992)

10. De Campos, C.P., Zeng, Z., Ji, Q.: Structure learning of Bayesian networks using constraints. In: Proceedings of the 26th Annual International Conference on Machine Learning, pp. 113-120. ACM (2009)

11. Duda, R.O., Hart, P.E.: Pattern Classification and Scene Analysis. Wiley, New York (1973)

12. Erkut, E.: The discrete p-dispersion problem. Eur. J. Oper. Res. 46(1), 48-60 (1990)

13. Fillbrunn, A., Berthold, M.R.: Diversity-driven widening of hierarchical agglomerative clustering. In: Fromont, E., Bie, T., Leeuwen, M. (eds.) IDA 2015. LNCS, vol. 9385, pp. 84-94. Springer, Heidelberg (2015). doi:10.1007/978-3-319-24465-5_8

14. Friedman, N., Geiger, D., Goldszmidt, M.: Bayesian network classifiers. Mach. Learn. 29(2-3), 131-163 (1997)

15. Golub, G.H., van Loan, C.F.: Matrix Computations, 4th edn. The Johns Hobpkins University Press, Baltimore (2013)

16. Hamming, R.W.: Error detecting and error correcting codes. Bell Syst. Tech. J. 29(2), 147-160 (1950)

17. Heckerman, D., Geiger, D., Chickering, D.M.: Learning Bayesian networks: the combination of knowledge and statistical data. Mach. Learn. 20(3), 197-243 (1995)

18. Ivanova, V.N., Berthold, M.R.: Diversity-driven widening. In: Tucker, A., Höppner, F., Siebes, A., Swift, S. (eds.) IDA 2013. LNCS, vol. 8207, pp. 223-236. Springer, Heidelberg (2013). doi:10.1007/978-3-642-41398-8_20

19. Koski, T.J., Noble, J.M.: A review of Bayesian networks and structure learning. Math. Applicanda 40(1), 53-103 (2012) 
20. Larrañaga, P., Karshenas, H., Bielza, C., Santana, R.: A review on evolutionary algorithms in Bayesian network learning and inference tasks. Inf. Sci. 233, 109-125 (2013)

21. Lichman, M.: UCI Machine Learning Repository (2013)

22. Lowerre, B.T.: The HARPY speech recognition system. Ph.D. thesis, Carnegie Mellon University, Pittsburgh, PA, USA (1976)

23. Maron, M.E., Kuhns, J.L.: On relevance, probabilistic indexing and information retrieval. J. ACM (JACM) 7(3), 216-244 (1960)

24. Meinl, T.: Maximum-score diversity selection. Ph.D. thesis, University of Konstanz, July 2010

25. Nielsen, J.D., Kočka, T., Peña, J.M.: On local optima in learning Bayesian networks. In: Proceedings of the Nineteenth Conference on Uncertainty in Artificial Intelligence, pp. 435-442. Morgan Kaufmann Publishers Inc., San Francisco (2003)

26. Pearl, J.: Probabilistic Reasoning in Intelligent Systems: Networks of Plausible Inference. Morgan Kaufmann Publishers Inc., San Francisco (1988)

27. Pernkopf, F.: Bayesian network classifiers versus k-NN classifier using sequential feature selection. In: AAAI, pp. 360-365 (2004)

28. Robinson, R.W.: Counting unlabeled acyclic digraphs. In: Little, C.H.C. (ed.) Combinatorial Mathematics V. LNM, vol. 622, pp. 28-43. Springer, Heidelberg (1977)

29. Sampson, O., Berthold, M.R.: Widened KRIMP: better performance through diverse parallelism. In: Blockeel, H., Leeuwen, M., Vinciotti, V. (eds.) IDA 2014. LNCS, vol. 8819, pp. 276-285. Springer, Heidelberg (2014). doi:10.1007/ 978-3-319-12571-8_24

30. Schwarz, G.: Estimating the dimension of a model. Ann. Stat. 6(2), 461-464 (1978)

31. Sierra, B., Larrañaga, P.: Predicting the survival in malignant skin melanoma using Bayesian networks. an empirical comparison between different approaches. Artif. Intell. Med. 14(1-2), 215-230 (1998)

32. Sprites, P., Glymour, C., Scheines, R.: Causation, Prediction, and Search. MIT Press, Cambridge (1993)

33. Jiang, S., Zhang, H.: Full Bayesian network classifiers. In: Proceedings of the 23rd International Conference on Machine Learning, pp. 897-904. ACM (2006)

34. Suzuki, J.: A construction of Bayesian networks from databases based on an MDL principle. In: Proceedings of the Ninth International Conference on Uncertainty in Artificial Intelligence, pp. 266-273. Morgan Kaufmann Publishers Inc., San Francisco (1993)

35. Tsamardinos, I., Brown, L.E., Aliferis, C.F.: The max-min hill-climbing Bayesian network structure learning algorithm. Mach. Learn. 65(1), 31-78 (2006) 